\title{
Silkworm Immunity and Hormonal Regulation
}

\section{Abir A Gad* \\ Department of Applied Entomology and Zoology, Egypt}

*Corresponding author: Abir Abd El Mageid Gad, Department of Applied Entomology and Zoology, Alexandria University, Faculty of Agriculture (Elshatby), Egypt, Tel: 002-01111450311; Email: Abir_gad@yahoo.com

\section{Review Article \\ Volume 2 Issue 1}

Received Date: December 20, 2018

Published Date: January 17, 2019

DOI: $10.23880 /$ izab-16000134

\section{Abstract}

Silkworm diseases are the most important disease that inflects heavy loss to crops. Most losses in sericulture can be attributed directly to silkworm diseases.

In insects, it relies on both humeral and cellular responses that are mediated via certain recognizing receptors and activation of several pathways. Fat body and hemocytes are the origins for the production and secretion of antimicrobial agents and activators/regulators of cellular response, while cell mediated immunity in insects is performed by hemocytes. Humoral immunity acts as a front-line barrier against invading pathogens. The humoral response includes, the activation of the Phenol oxidase system, which triggers the synthesis of melanin and ii) the production of several immune effectors, such as lysozyme, reactive oxygen and nitrogen species, and antimicrobial peptides (AMPs).

Keywords: Humoral Immunity; Hemocytes; Haemolymph

Abbreviations: AMPs: Antimicrobial Peptides; PO: Prophenoloxidase; Ddc: Dopa Decarboxylase; FAAs: Free Amino Acids; JH: Juvenile Hormone; CA: Corpora Allata; NVP: Nucleopolyhedrovirus; ICP: Insecticidal Crystal Protein; BB: Bacillus Bombysepticus; BT: Bacillus Thuringiensis.

\section{Introduction}

\section{Cellar Immunity}

Insect haemolymph contains several types of mesodermal cells that are suspended in plasma, attached to various organs or attached to the inner surface of integument. Circulating haemocyte, carry out an immune response, or cellular defense, via phagocytosis, nodulation, encapsulation and melanization.

These processes appear to be discrete immune responses in terms of gene expression and outcome.
However, these certain immune responses share a number of common elements that function in concert to clear pathogens from the hemolymph.

Phagocytosis is induced when phagocyte surface receptors, are activated by target cells. It must be noted that the hemocyte response to various bacteria differs. For example, in $A$. aegypti hemocytes respond to Escherichia coli with phagocytosis, whereas to Micrococcus luteus with melanization [1-5].

Gad and Alzahofi [6] explained that hyperphagocytic hemocytes are involved in nodule formation and phagocytosis to clear large numbers of bacteria at the early stages of infection, while plasmatocytes and granulocytes are involved in phagocytosis of bacterial cells remained in circulation. Also, demonstrated that the immune response against bacterium is the same in pathogenic and non pathogenic bacteria. 


\section{International Journal of Zoology and Animal Biology}

\section{Nodulation}

Nodulation refers to multicellular hemocytic aggregates that entrap a large number of bacteria. Melanized or non-melanized nodules are formed in response to a number of invaders. Nodule formation appears to be related with eicosanoids in many insect species [7] or prophenoloxidase (PO) and dopa decarboxylase (Ddc) in medfly hemocytes [8].

\section{Encapsulation}

Encapsulation refers to the binding of hemocytes to larger targets, such as parasites, protozoa, and nematodes. Encapsulation can be observed when parasitoid wasps lay their eggs in the hemocel of Drosophila larvae. Hemocytes after binding to their target they form a multilayer capsule around the invader, which is ultimately accompanied by melanization. Within the capsule the invader is killed, by the local production of cytotoxic free radicals ROS and RNS, or by asphyxiation $[5,9]$.

\section{Humoral Immunity}

Humoral immunity acts as a front-line barrier against invading pathogens, and the majority of the components are widely conserved among species. Regulation of innate immunity is important for overcoming infections and preventing self-damaging sepsis.

Defense peptides are key factors in humoral immunity against bacteria and fungi in invertebrates. Antimicrobial peptides play a crucial role in fighting against invading pathogens .They are synthesized in response to microbial infection or septic body injury mainly in insect fat body and in certain blood cells, and then rapidly released into the haemolymph where they act synergistically against microorganisms [10]. Anti-microbial peptide homologous to $B$. mori, cecropin $\mathrm{D}$ and a proline-rich peptide of unique amino acid sequence were purified by Morishima, et al. [11].

To understand the role of such network of amino acids in the defense mechanism against bacterial infection, it is important to know about many antibacterial peptides which were isolated from different species of insects. Such peptides can be classified into five major groups: Cecropins, insect defensins, attacin-like protein, proline rich peptides and lysozyme.

In B.mori, cecropins and lysozyme have been reported as antibacterial peptides by Abraham, et al. [12] According to Hara and Yamakawa, et al. [13] insect defenses are highly effective against Gram-positive bacteria, including pathogenic bacteria such as
Staphylococcus aureus, whereas they do not exhibit strong activity against gram negative bacteria. Also, they isolated antibacterial peptide from B.mori larvae which was immunized with gram-positive bacteria Staphylococcus aureus called moricin that consists of 42 amino acids.

Some amino acid sequences of the moricin peptide are as follows: Lys-Ala-Ile-Asp-Gly- Val-Arg-Leu-Phe-Pro-ThrHis-Ser. Moricin is believed to have antibacterial activity against both gram negative and some pathogenic positive bacteria such as Bacillus cereus. Furthermore, [13] isolated three antibacterial peptides from B.mori larvae which were immunized with gram-negative bacteria E. coli. These peptides were 32 amino acids long and characteristically rich in proline residues. Some of amino acid of the novel peptides was Pro, Leu, Tyr, Arg, Asp, Lys, IIe, Gly, Phe, Met, Val and Thr. These peptides are effective against gram-negative bacteria such as: E. coli and Acinetobacter sp.

Gad et al. [14] evaluated the effect of Escherichia coli and Bacillus thuringeinsis infection on amino acids in the fifth larval instar of B. mori, 16 free amino acids (FAAs) were observed, these amino acids were: Aspartic acid, Threonine, Serine, Glutamic acid, Proline, Glycine, Alanine, Valine, Methionine, Isoleucine, leucine, Tyrosine, Phenylalanine, Histidine, Lysine and arginine in the control and treatment. There was a marked increase in some FAAs, eg: Threonine, Serine, Proline, Glycine, Valine, Methionine, leucine, Phenylalanine, Histidine, Lysine and arginine after 24 and $48 \mathrm{hrs}$. These FAAs formed the major sequences in moricin and other novel antibacterial peptides [13]. On the other hand, the concentrations of aspartic, glutamic, glycine, alanine, tyrosine and lysine in the haemolymph of immunized larvae were relatively low. These amino acids are six of the known eight major FAAs forming a fibroin molecule of the silk filament [15].

These results can be explained that the larval metabolism was busy exhibiting immunological responses against bacterial infection producing anti- bacterial peptides in addition to cellular immunity while silk protein was greatly decreased.

\section{The Hormonal Regulation of Silkworm Immunity}

Insect humoral immunity can be affected by juvenile hormone $(\mathrm{JH})$ and 20-hydroxyecdysone (20E), but how humoral immunity is developmentally regulated by these two hormones in insects and has not yet been elucidated. Fat body produces humoral response molecules and hence is considered as the major organ involved in innate immunity [16]. Tian, et al. [17] suggested that JH plays a 


\section{International Journal of Zoology and Animal Biology}

positive role in the regulation of innate immunity in the larval fat body. Also, Riddiford, et al. [18] suggested that $\mathrm{JH}$ has a significant role in the control of immune humoral function. A number of studies in Drosophila imply that 20E induces AMP mRNA expression and acts as an immuneactivator Silverman, et al. [19] while $\mathrm{JH}$ acts as an immune-suppressor by antagonizing 20E signaling [20]. Also, Flatt, et al. [21] found that juvenile hormone regulate the immunity of Drosophila by inhibiting phenoloxidase (Po) synthesis and prevents cuticular melanization.

Gad, Abdel-Megeed, et al. [22] demonstrated that injection of B. mori fifth larval instar with B. thuringiensis caused a sharp decrease in the corpora allata (CA) surface area up to $96 \mathrm{~h}$ post-infection to reach the minimum value of about $54.5 \%$ less than the control which is followed by a marked increase in the CA surface area at $120 \mathrm{~h}$ about $12.18 \%$ over the control. This result may due to the effect of bacteria on CA activity during a few days of infection and the increase of the CA activity after that may be due to the release of antibacterial peptides as an immunity response against bacteria which helped the gland to reprogrammed itself in its cycle during the last larval instar. The corpora allata (CA) volume was used as an indicator of the juvenile hormone (JH) level [23].

\section{Mutagenic Effects of Pathogenic Bacteria on Silkworm}

It is important to explain the side effects and the mode of action by which the injection of the bacteria can affect the physiology and genetics of $B$. mori.

A molecular marker of B. mori DNA is the most important method for determining the affected region on DNA since they reveal DNA polymorphisms among genetically related individuals [24]. A similar strategy has been used to identify the nucleopolyhedrovirus (NPV), another important silkworm genotype virus [25]. B. thuringiensis is the most widely used microbial pesticides. The biochemical basis of the pesticide is an insecticidal crystal protein (ICP), which is produced by the bacterium as a 133-kDa protoxin that requires proteo-lytic cleavage in the insect gut for activation. The muta-genic effect of the bacterial injection produced several proteins that enable it to kill insects through the alteration of the physiological processes [26].

Gad, Abdel-Megeed, wt al. [22] observed that injection of B. mori fifth larval instar with B. thuringiensis and E. coli caused genotoxicity in B. mori DNA band region, which composed of several structural domains that are disrupted by the toxin secreted by both B. thuringeinsis and E. coli against B. mori.

Huang, et al. [27] suggested that injection of B. mori with Bacillus bombysepticus (Bb) caused a lot of basal metabolic pathways which were significantly modulated. Furthermore, genes of juvenile hormone synthesis and related metabolism showed up regulation, suggesting that juvenile hormone participate in host modulation during the infection. Moreover, host cellular and systemic immune responses are also induced. Similar to $B$. thuringiensis (Bt), $\mathrm{Bb}$ can also induce a silkworm poisoning-related response.

\section{References}

1. Hernandez Martinez S, Lanz $\mathrm{H}$, Rodriguez $\mathrm{MH}$, Gonzalez Ceron L, Tsutsumi V (2002) Cellularmediated reactions to foreign organisms inoculated into the hemocoel of Anopheles albimanus (Diptera: Culicidae). J Med Entomol 39(1): 61-69.

2. Hillyer JF, Schmidt SL, Christensen BM (2003a) Hemocyte-mediated phagocytosis and melanization in the mosquito Armigeres subalbatus following immune challenge by bacteria. Cell Tissue Res 313(1): 117-127.

3. Hillyer JF, Schmidt SL, Christensen BM (2003b) Rapid phagocytosis and melanization of bacteria and Plasmodium sporozoites by hemocytes of the mosquito Aedes aegypti. J Parasitol 89(1): 62-69.

4. Schmidt 0, Theopold, U, Strand M (2001) Innate immunity and its evasion and suppression by hymenopteran endoparasitoids. BioEssays 23(4): 344-351.

5. Tsakas S, Marmaras VJ (2010) Insect immunity and its signalling: an overview. ISJ 7: 228-238.

6. Gad A Abir, Nora N Alzahofi (2009) Changes in the haemocytes of Bombyx mori larvae (Lepidoptera: Bombycidae) in relation to Bacillus thuringensis infection. The Egypt Sci Magaz 6(1-2): 15-22.

7. Miller JS, Howard RW, Rana RL, Tunaz H, Stanley DW (1999) Eicosanoids mediate nodulation reactions to bacterial infections in adults of the cricket, Gryllus assimilis. J Insect Physiol 45(1): 75-83.

8. Sideri M, Tsakas S, Markoutsa E, Lampropoulou M, Marmaras VJ (2008) Innate immunity in insects: surface-associated dopa decarboxylase-dependent 


\section{International Journal of Zoology and Animal Biology}

pathways regulate phagocytosis, nodulation and melanization in medfly haemocytes. Immunology 123(4): 528-537.

9. Nappi AJ, Vass E, Frey F, Carton Y ( 2000) Nitric oxide involvement in Drosophila immunity. Nitric Oxide 4(4): 423-430.

10. Jarosz J (1995) Haemolymph immune proteins protect the insect body cavity from invading bacteria. Comp Biochem Physiol 111(2): 213-220.

11. Morishima I, Horiba T, Iketani M, Nishioka E, Yamano Y (1995) Parallel induction of cecropin and lysozyme in larvae of the silk worm. Dev Comp Immunol 19(5): 357-363.

12. Abraham E, Nagaraju J, Salunke D, Gupta HM, Datta RK (1995) Purification and Partial Characterization of an Induced Antibacterial Protein in the Silkworm, Bombyx mori. J Invertebr Pathol 65(1): 17-24.

13. Hara S, Yamakawa M (1995) Moricin, a Noval Type of Antibacterial Peptide Isolated from the silkworm, Bombyx mori. The journal of Biological. Chemistry 270(50): 29923-29927.

14. Gad A Abir (2012) Immune responses of Bombyx mori larvae towards the bacterial infections with Escherichia coli and Bacillus thuringeinsis. The Egypt Sci Magaz 7(3-4): 41-45.

15. Principle and Practices Serriculture (1990) A book published by National Serriculture and Entomology Research Institute, Rural Development Administration, Republic of Korea, pp: 539.

16. Muramatsu D, Kinjoh T, Shinoda T, Hiruma K (2008) The role of 20-hydroxyecdysone and juvenile hormone in pupal commitment of the epidermis of the silkworm, Bombyx mori. Mech Dev 125(5-6): 411420.

17. Tian L, Enen G, Yupu D, Shun Z, Qin P, et al. (2010) Genome-wide regulation of innate immunity by juvenile hormone and 20-hydroxyecdysone in the Bombyx fat body. BMC Genomics 11: 549.

18. Riddiford LM (1994) Cellular and molecular actions of juvenile hormone. I. General considerations and premetamorphic actions. Advance Insect Physiol 24: 213-274.
19. Meister M, Lagueux M (2003) Drosophila blood cells. Cell Microbiol 5(9): 573-580.

20. Flatt T, Heyland A, Rus F, Porpiglia E, Sherilock C, et al. (2008) Hormonal regulation of the humoral innate response in Drosophila melanogaster. J Exp Biol 211(16): 2712-2724.

21. Flatt T, Tu MP, Tatar M (2005) Hormonal pleiotropy and the juvenile hormone regulation of Drosophila development and life his-tory. Bioessays 27(10): 9991010.

22. Gad A Abir, Abdel Megeed AA (2013) Physiological and mutagenic effects of Pathogenic and non pathogenic bacteria on the last larval instar of Bombyx mori (Lepidoptera: Bombycidae). African journal of microbiology research 7(48): 5433-5439.

23. Pflugefelder O (1948) Volumetrissche untersuchugen van den corpora allata der Honigbiene Apis mellifera. Biol Zbl 66: 211-235.

24. Williams JG, Kubelik AR, Livak KJ, Rafalski JA (1990) DNA polymorphisms amplified by arbitrary primers are useful as genetic markers. Nucleic Acids Res 18(22): 6531-6535.

25. Yao Q, Lim W, Wang Y, Wang WB, Lu J, et al. (2003) Screening of molecular markers for NPV resistance in Bombyx mori L. (Lep, Bombycidae). J Appl Entomol 127(3): 134-136.

26. Brown SE, Cao AT, Eric R, Hines N, Akhurst RJ, et al. (2004) A novel secreted protein toxin from the insect pathogenic bacterium Xenorhabdus nematophila. J Biol Chem 279(15): 14595-14601.

27. Huang L, Tingcai C, Pingzhen X, Daojun C, Ting F, et al. (2009) A Genome-Wide Survey for host Response of silkworm, Bombyx mori during pathogen Bacillus bombyseptieus infection. PLoS One 4(12): e8098.

28. Gillespie JP, Kanost MR, Trenczek T (1997) Biological mediators of insect immunity. Annu Rev Entomol 42: 611-643.

29. Lamprou I, Tsakas S, Theodorou GL, Karakantza M, Lampropoulou M, et al. (2005) Uptake of LPS/E. coli/latex beads via distinct signalling pathways in medfly hemocytes: the role of MAP kinases activation and protein secretion. Biochim Biophys Acta 1744(1): 1-10. 